\title{
Retinal Nerve Fiber Layer Thickness and Macular Ganglion Cell Evaluation in Unilateral Amblyopia using Optical Coherence Tomography H.M.El-Fiky ${ }^{1}$, A.A.Fayed ${ }^{1}$ and M.A.El-Nagar ${ }^{2}$ \\ ${ }^{1}$ Ophthalmology Dept., Faculty of Medicine, Benha Univ., Benha, Egypt \\ ${ }^{2}$ Ophthalmology Dept., Benha Teaching Hospital, Benha, Egypt \\ E-Mail: dr.mohamed.elnagar85@gmail.com
}

\begin{abstract}
The aim of the current study is to evaluate retinal nerve fiber layer (RNFL) thickness, macular parameters and ganglion cell thickness (GCL+) in unilateral anisometropic amblyopic eye in relation to the normal healthy fellow eye and control one using spectral domain optical coherence tomography (SD-OCT). This study included 56 eyes of 28 cases aged from 8 30 years old subdivided into: 20 amblyopic eyes of patients with unilateral anisometropic amblyopia (group A), 20 sound fellow eyes of the unilateral anisometropic amblyopic patients (group B) and 16 emmetropic eyes of normal non amblyopic control (group C). Anisometropic amblyopic patients further subdivided according to refractive error into hypermetropic, myopic, and astigmatic amblyopic subgroups. All cases underwent detailed ophthalmic examination including visual acuity by Snellen's chart, cycloplegic refraction, ocular motility and alignment test, slit lamb biomicroscopy, intraocular pressure, fundus examination and OCT imaging by SD-OCT. Regarding peripapillary RNFL thickness, macular parameters including (central macular thickness (CMT), average macular thickness and macular volume), and GCL+ thickness, there was no significant difference among the amblyopic, fellow eyes, and normal eyes of non-amblyopic group, $\mathrm{P}>0.05$. By further analysis to compare subgroups according to the refractive error between amblyopic and their fellow eyes, peripapillary RNFL thickness revealed a significant increase in amblyopic eyes of hypermetropic (mean $121.750 \pm 8.647 \mu \mathrm{m})$ and astigmatic subgroups (mean $111.888 \pm 7.043 \mu \mathrm{m})$ compared with their fellow eyes $(116.125 \pm 8.509$ and 107.333 \pm 6.480 ) respectively, but it was significantly thinner in myopic amblyopic eyes (mean $81.333 \pm 23.352 \mu \mathrm{m})$ than that of their fellow eyes (mean $110.00 \pm 17.578$ ). CMT revealed a significant increase in myopic amblyopic eyes (mean $199.00 \pm 26.851 \mu \mathrm{m}$ ) compared with their fellow eyes (mean 191.00 $\pm 26.00 \mu \mathrm{m}$ ). Other OCT parameters did not significantly differ between amblyopic and their fellow eyes in different subgroups. OCT parameters including peripapillary RNFL thickness, macular parameters, and GCL+ thickness showed no significant difference among the amblyopic, fellow eyes, and normal eyes of non-amblyopic group. Hypermetropic and astigmatism amblyopic eyes showed increased peripapillary RNFL thickness than their fellow eyes, while myopic amblyopic eyes had decreased peripapillary RNFL thickness than their fellow eyes. CMT revealed a significant increase in amblyopic eyes of the myopic anisometropic subgroup compared with their fellow eyes.
\end{abstract}

Keywords: Amblyopia, Spectral domain optical coherence tomography.

\section{Introduction}

Amblyopia is a developmental ocular disorder characterized by a decrease in best corrected visual acuity (BCVA) to less than 6/9 of snellen's optotype in one or both eyes or by two or more lines difference in Logarithm of minimum angle of resolution (Log Mar ) optotype between two eyes at first years of life. It occurs due to abnormal visual experience during critical period of visual development, with no ocular abnormalities [1, 2].

It is considered a socioeconomic disorder with a prevalence of 2-4 \% of general population [3].

Amblyopia can be classified into stimulus deprivational, strabismic, anisometropic, and bilateral ametropic amblyopia [4]. Amblyopia mainly result from abnormal visual stimulation early in life which induces cell shrinkage and anatomical changes in the LGN layers and visual striate cortex connected with amblyopic eye, and therefore, cells of the primary visual cortex lose their ability to respond to stimulation of amblyopic eye [5].

Optical coherence tomography (OCT) is a noninvasive and non-contact technique that provides high- resolution cross sectional imaging of retinal layer. With the advances in OCT technique, it is possible to get more detailed information about retinal involvement in amblyopia. The use of SD-OCT has become the mainstream, as it has better spatial resolution and higher scan speed than time domain optical coherence tomography (TD-OCT) types [6].

Many studies examined the amblyopic retinal thickness using OCT and the degree of retinal involvement accompanying amblyopia is controversial. Some of these studies reported no differences between amblyopic and their healthy normal fellow eyes [6-10], whereas others reported significant differences between amblyopic and healthy eyes [11-15].

The aim of this study was to investigate peripapillary RNFL thickness, macular parameters, and GCL+ thickness in amblyopic eyes in comparison to their normal fellow eyes and normal eyes of non-amblyopic cases.

\section{Subjects and methods \\ 2.1 Subjects}

The study was performed at the outpatient clinic of ophthalmology hospital, Benha University, as a prospective comparative highly selective cross-sectional study from July 2017 to May 2020. Informed consent for the examinations was obtained from each patient or one of their parents.

The study included 56 eyes of 28 cases aged 8-30 years old subdivided into 3 groups, Group A: included 20 amblyopic eyes of patients with unilateral anisometropic amblyopia, Group B: included 20 sound 
fellow eyes of the anisometropic amblyopic patients, and Group C: included 16 emmetropic eyes of normal non amblyopic control.

Inclusions criteria: Amblyopic patients and control non amblyopic subjects aged from 8 to 30 years old., unilateral anisometropic amblyopic patients with BCVA difference of $\geq 2$ lines on Snellen's chart between the amblyopic and fellow eyes, anisometropia with cycloplegic spherical equivalent $(\mathrm{SE})$ difference $\geq 2.0$ diopters between the two eyes, the BCVA of fellow sound eyes and normal eyes of non-amblyopic subjects were $\leq 0.1 \log$ MAR.

Exclusion criteria: Systemic disease as diabetes mellitus and hypertension, neurological disorders, ocular disease that may affect RNFL (e.g. Diabetic retinopathy), optic nerve disease such as glaucoma, ocular media opacities (e.g. corneal opacity, or cataract), nystagmus, history of ocular surgery, and previously treated amblyopic patients.

\subsection{Methods}

All subjects enrolled in this study underwent all ophthalmologic examination including visual acuity assessment by Snellen's chart, cycloplegic refraction, ocular motility test, cover-uncover test and alternate cover test, slit lamb examination of anterior segment, intraocular pressure, fundus examination, and OCT measurement.

All OCT scans were performed using a spectral domain OCT (Topcon 3D OCT-2000 FA, Japan "version 8.30.) with infrared [ $840 \mathrm{~nm}]$ and $<5 \mu \mathrm{m}$ axial resolution for measuring peripapillary RNFL thickness, central macular thickness (CMT), average macular thickness, macular volume, and ganglion cell layer plus inner plexiform layer (GCL+) thickness.

\subsection{Statistical methods}

All statistical analysis of the collected data were performed using Statistical Package for Social Science (SPSS), IBM SPSS Statistics for Windows, and Version 25.0. Armonk, NY: IBM Corp. Statistical methods were used to analyze the results of the present study depend on the type of obtained data. Qualitative data were described as number and percentage. Quantitative data were described using

arithmetic mean with standard deviation (SD) as mean \pm $\mathrm{SD}$, and range. One-way ANOVA followed by Tukey's test were used to compare the OCT parameters among 3 main groups (Amblyopic "group A", fellow "group B" and normal control eyes "group C"). Paired t-test was used to assess the statistical significance and compare between the two eyes of amblyopic patients (amblyopic eyes and their fellow eyes) in subgroup analysis. In all statistical tests, level of significance of 0.05 was used, Pvalues $<0.05$ were considered to be statistically significant and vice versa.

\section{Results}

The current study was conducted on 56 eyes of 28 cases subdivided as: 20 anisometropic amblyopic eyes of patients with unilateral anisometropic amblyopia (group A), 20 sound fellow eyes of the amblyopic patients (group B), and 16 emmetropic eyes of non-amblyopic control (group C). The mean age $\pm \mathrm{SD}$ of amblyopic cases was $18.35 \pm 8.87$ years old, and the mean age of normal control cases was $20.40 \pm 7.50$ years old, $40 \%$ males and $60 \%$ female of group(A) and (B), 37\% male and 63\% female of $\operatorname{group}(\mathrm{C})$.

Mean BCVA by $\log$ MAR were $0.757 \pm 0.169$ for group (A), $0.108 \pm 0.090$ for group (B), and $0.00 \pm 0.00$ for group $(\mathrm{C})$.

By further subdivision of 20 amblyopic eyes according to SE refractive error, 8 were hypermetropic amblyopic eyes, 3 were myopic amblyopic eyes, and 9 were astigmatic amblyopic eyes. The mean BCVA by $\log$ MAR in hypermetropic amblyopic subgroup were $0.748 \pm 0.152$ and $0.157 \pm 0.063$ in the amblyopic and fellow eyes, respectively, in myopic amblyopic subgroup were $0.867 \pm 0.231$ and $0.060 \pm 0.104$ in the amblyopic and fellow eyes, respectively, and in astigmatic amblyopic subgroup were $0.729 \pm 0.172$ and $0.080 \pm 0.095$ in the amblyopic and fellow eyes, respectively. A significant differences were found between amblyopic and fellow eyes in the 3 subgroups regarding BCVA.

No significant difference was observed among group (A), (B), and (C) regarding peripapillary RNFL thickness, CMT, average macular thickness, macular volume and GCL+ thickness Table(1).

With comparing the amblyopic eyes versus the fellow eyes according to SE refractive error Table(2), an increased peripapillary RNFL was observed in both the hypermetropic amblyopic eyes and astigmatic amblyopic eyes compared with their fellow, (P-value $<0.05)$, whereas myopic amblyopic subgroup showed a significantly thinner peripapillary RNFL in the amblyopic eyes than their fellow eyes $(\mathrm{P}=0.044)$ Fig $(1)$.

With comparing CMT, no significant difference were detected in both hypermetropic and astigmatic subgroups of the amblyopic eyes compared with their fellow eyes, while the CMT was found to be significantly thicker in myopic amblyopic eyes than their fellow eyes $(\mathrm{P}=0.005)$ Fig (2). Regarding GCL+ thickness, no significant difference were detected between amblyopic and their sound fellow eyes in any anisometropic subgroups ( $\mathrm{p}$ value $>0.05$ ) Fig (3).

\section{Discussion}

Amblyopia may cause some anatomic alterations in the retina. OCT offered reliable and accurate crosssectional imaging of the retinal structure and evaluation of the retinal changes accompanying amblyopia. However, evidences for direct retinal changes in amblyopia remain controversial $[9,16]$. In the current study we compared the peripapillary RNFL thickness, macular parameters, and GCL+ thickness of amblyopic eyes (group A) with that of fellow normal eyes (group B) and normal control eyes of non-amblyopic subjects (group C) using SD-OCT.

Table (1) Comparison between group (A),(B),and(C) regarding the OCT parameters. 


\begin{tabular}{|c|c|c|c|c|}
\hline OCT parameter & $\begin{array}{c}\text { Group (A) } \\
(\text { mean } \pm \text { SD) }\end{array}$ & $\begin{array}{c}\text { Group (B) } \\
(\text { mean } \pm \text { SD) }\end{array}$ & $\begin{array}{c}\text { Group (C) } \\
(\text { mean } \pm \text { SD) }\end{array}$ & P-value \\
\hline \multicolumn{5}{|l|}{ Peripapillary RNFLT $(\mu \mathrm{m})$} \\
\hline Superior & $130.05 \pm 18.729$ & $130.05 \pm 12.378$ & $126.533 \pm 13.399$ & 0.749 \\
\hline Inferior & $132.30 \pm 27.654$ & $137.30 \pm 15.898$ & $136.20 \pm 17.407$ & 0743 \\
\hline Average & $111.10 \pm 17.165$ & $111.25 \pm 9.716$ & $108.80 \pm 10.625$ & 0.837 \\
\hline \multicolumn{5}{|l|}{ Macular parameter } \\
\hline $\mathrm{CMT}(\mu \mathrm{m})$ & $191.50 \pm 16.863$ & $191.35 \pm 16.887$ & $187.40 \pm 12.603$ & 0.704 \\
\hline Average macular thickness $(\mu \mathrm{m})$ & $271.46 \pm 14.725$ & $274.61 \pm 11.854$ & $275.32 \pm 7.201$ & 0.586 \\
\hline Total macular volume $\left(\mathrm{mm}^{3}\right)$ & $7.712 \pm 0.427$ & $7.795 \pm 0.269$ & $7.778 \pm 0.205$ & 0.698 \\
\hline Average GCL+ $(\boldsymbol{\mu m})$ & $69.05 \pm 10.092$ & $71.75 \pm 4.876$ & $73.4 \pm 2.197$ & 0.173 \\
\hline
\end{tabular}

RNFLT, retinal nerve fiber layer thickness; CMT, central macular thickness; GCL+, ganglion cell layer plus inner plexiform layer; OCT, optical coherence tomography.

Table (2) Comparison between amblyopic and fellow eyes of refractive subgroups regarding the OCT parameters.

\begin{tabular}{|c|c|c|c|}
\hline OCT parameter & $\begin{array}{c}\text { Amblyopic eyes } \\
(\text { mean } \pm \text { SD })\end{array}$ & $\begin{array}{l}\text { Fellow eyes } \\
(\text { mean } \pm \text { SD })\end{array}$ & P-value \\
\hline \multicolumn{4}{|l|}{ Average peripapillary RNFL ( $\mu \mathrm{m})$} \\
\hline hypermetropia & $121.750 \pm 8.647$ & $116.125 \pm 8.509$ & $0.002 * *$ \\
\hline myopia & $81.333 \pm 23.352$ & $110.00 \pm 17.578$ & $0.044 *$ \\
\hline Astigmatism & $111.888 \pm 7.043$ & $107.333 \pm 6.480$ & $0.035^{*}$ \\
\hline \multicolumn{4}{|l|}{ CMT $(\mu \mathrm{m})$} \\
\hline hypermetropia & $184.125 \pm 9.372$ & $191.00 \pm 26.000$ & 0.072 \\
\hline myopia & $199.00 \pm 26.851$ & $191.00 \pm 26.000$ & $0.005 * *$ \\
\hline Astigmatism & $195.555 \pm 18.007$ & $188.555 \pm 14.248$ & 0.132 \\
\hline \multicolumn{4}{|l|}{ Average GCL+ $(\mu \mathrm{m})$} \\
\hline hypermetropia & $74.125 \pm 4.015$ & $73.750 \pm 4.166$ & 0.598 \\
\hline myopia & $50.666 \pm 14.153$ & $65.00 \pm 5.567$ & 0.257 \\
\hline Astigmatism & $70.666 \pm 4.716$ & $72.222 \pm 3.492$ & 0.071 \\
\hline
\end{tabular}

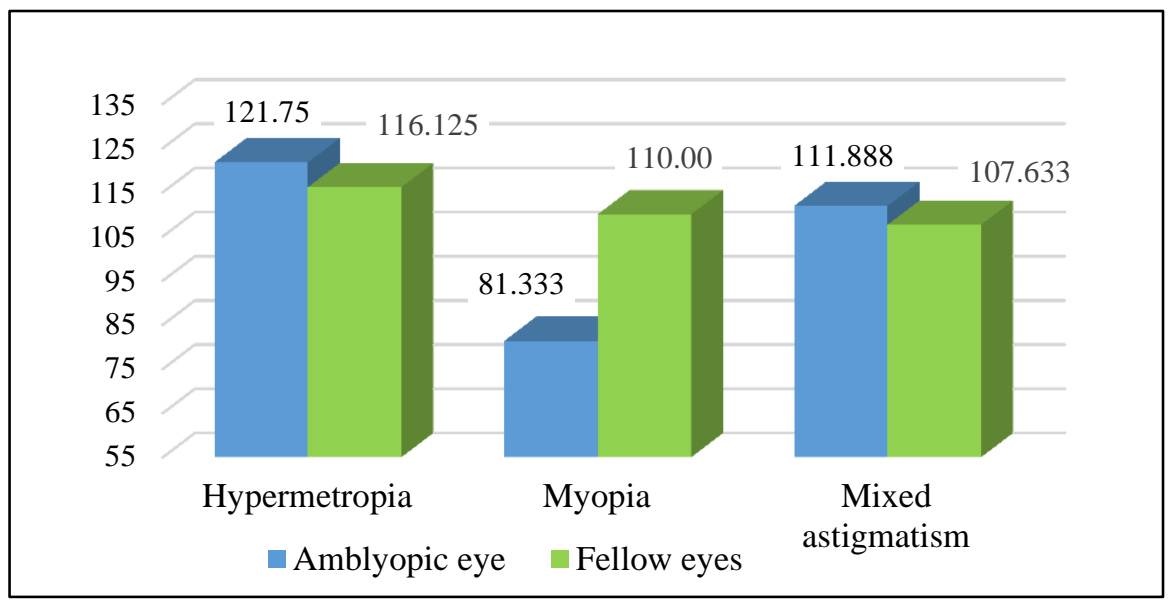

Fig (1) Bar chart indicating the comparison between amblyopic eyes and their fellow eyes in the three groups of anisometropia regarding average peripapillary RNFL thickness.

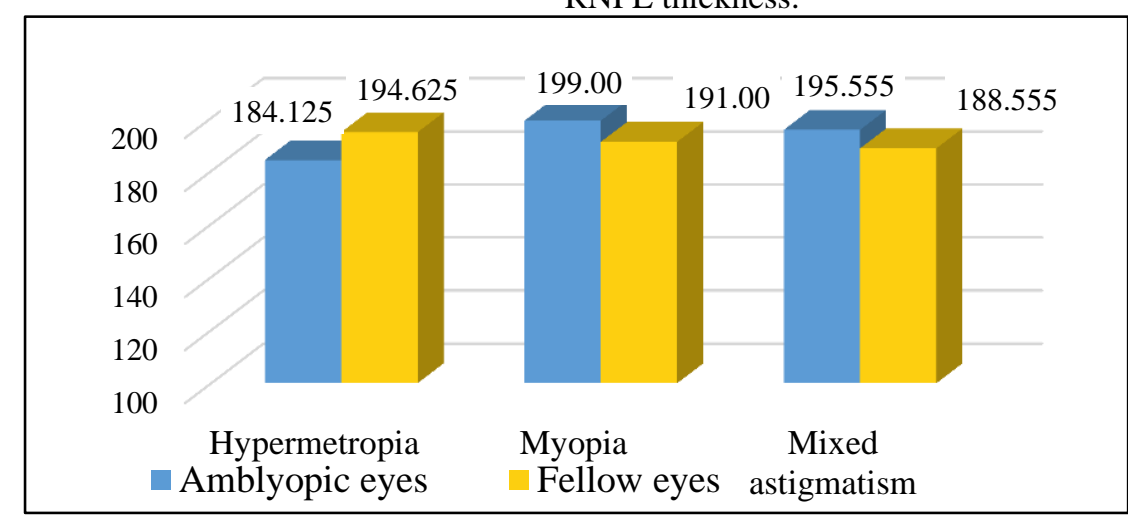


Fig (2) Bar chart indicating the comparison between amblyopic eyes and their fellow eyes in the three subgroups of anisometropia regarding CMT.

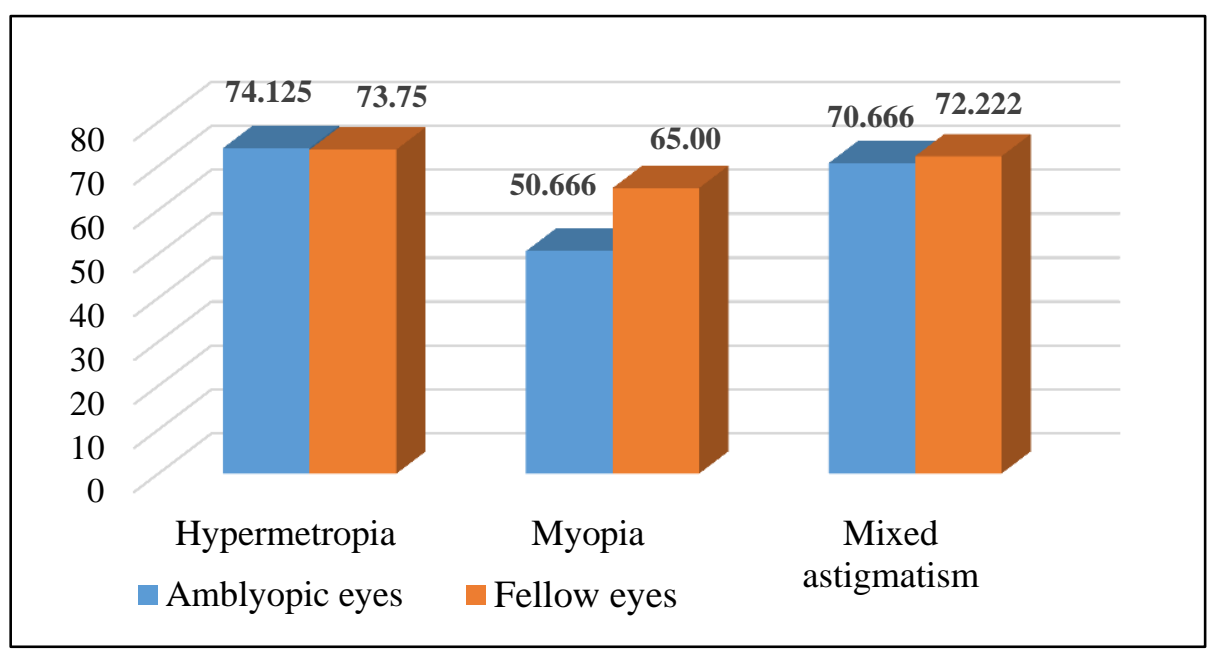

Fig (3) Bar chart indicating the comparison between amblyopic eyes and their fellow eyes in the three subgroups of anisometropia regarding GCL+ thickness.

This comparison revealed no significant difference in all OCT parameter among group $\mathrm{A}, \mathrm{B}$, and $\mathrm{C}$. The results of this study corroborate a previous OCT studies. Wang and Taranath concluded 14 patients aged 5-10 years with hyperopic anisometropic amblyopia and found no significant difference between amblyopic and their fellow eyes regarding peripapillary RNFL thickness, CMT, and macular volume. The mean of RNFL thickness was $103.4 \pm 12.0 \mu \mathrm{m}$ and $103.4 \pm 17.2 \mu \mathrm{m}$ in amblyopic and fellow eyes, respectively. The mean of macular thickness was $256.2 \pm 26.8 \mu \mathrm{m}$ and $255.1 \pm 22.9 \mu \mathrm{m}$ and the mean of macular volume was $10.3 \pm 0.8$ and $10.2 \pm 0.7 \mathrm{~mm} 3$ in amblyopic and fellow eyes, respectively[7]. Thirty six patients with unilateral amblyopia including strabismic and anisometropic amblyopia (5-23 years old) and 32 emmetropic non amblyopic cases (4 -24 years old) were included in the study carried out by Firat and colleagues who reported that the mean peripapillary RNFL thickness, macular thickness, and GCC did not significantly differ among the eyes [8]. Additionally, the study by Abdulghaffar and colleagues concluded no significant difference in peripapillary RNFL, CMT, or GCL+ between amblyopic and their fellow eyes [16].

In contrast to the results yielded by the current study, other studies found a significant difference in the retinal layer thickness between amblyopic and normal eyes. In instances, Kasem and Badawi found a statistically significant increase in the CMT $(196.2 \pm 50.03 \mu \mathrm{m})$ and RNFL thickness $(97.00 \pm 11.60 \mu \mathrm{m})$ in the amblyopic eyes compared with their fellow eyes as the mean were $167 \pm 12.76 \mu \mathrm{m}$ of $\mathrm{CMT}$ and $78.50 \pm 13.05 \mu \mathrm{m}$ of peripapillary RNFL thickness in 64 patients aged 7-32 years old with unilateral amblyopia [15]. Similarly, ElHifnawy et al. included 20 patients aged $15-40$ years old with unilateral amblyopia and reported an increase in CMT in the amblyopic eyes $(288.65 \pm 22.61 \mu \mathrm{m})$ versus their fellow eyes $(281.1 \pm 22.6 \mu \mathrm{m})$. They found also an increase in the mean thickness of the RNFL of the amblyopic eyes $(33.03 \pm 3.816 \mu \mathrm{m})$ compared with their normal fellow eyes $(30.76 \pm 3.75 \mu \mathrm{m})$. In the other hand, they found statistically significant decrease in the thickness of the GCL+ of the amblyopic eyes $(36.38 \pm 5.27 \mu \mathrm{m})$ compared with their fellow eyes $(39.09 \pm 6.19 \mu \mathrm{m})$ only at the outer macular circle[14]. Alotaibi and Enazi enrolled 93 patients with unilateral amblyopia aged 5-12 years old and concluded that the mean RNFL thickness was significantly increased in the amblyopic eyes $(112,16 \mu \mathrm{m})$ versus the fellow eye (106 $\mu \mathrm{m})$, but the macular thickness revealed no statistically significant difference $(\mathrm{P}=0.195)[12]$. Park et al. reported a significant thinning of the GCL+ in the amblyopic eye than their fellow eyes in 20 unilateral amblyopic patients aged 4-19 years old, while no significant difference was obtained regarding total macular thickness between amblyopic and their fellow eyes [17]. Kavitha et al. enrolled 60 children aged 5-18 years old (30 anisometropic amblyopia patients and 30 non amblyopic subjects) and found the macular thickness was greater in amblyopic eyes $(286.9 \pm 6.52 \mu \mathrm{m})$ than their fellow eyes $(240 \pm 10.45 \mu \mathrm{m})$ and normal eyes of normal cases (239.8 $\pm 4.294 \mu \mathrm{m})$, while the difference in mean RNFL thickness between amblyopic eyes $(100.87 \pm 6.24 \mu \mathrm{m})$ and fellow eyes $(100.5 \pm 2.895 \mu \mathrm{m})$; and normal eyes of nonamblyopic cases $(98.63 \pm 4.723 \mu \mathrm{m})$ statistically not significant.

By applying further analysis in the present study to compare RNFL thickness in anisometropic amblyopic eyes versus the fellow eyes according to SE refractive error, a significant increase was observed in both hypermetropic amblyopic eyes $(121.75 \pm 8.65 \mu \mathrm{m})$ and astigmatic amblyopic eyes $(111.89 \pm 7.04 \mu \mathrm{m})$ compared with the fellow eyes of the same subgroup (116.12 \pm 8.51 $\mu \mathrm{m}$ and $107.33 \pm 6.48 \mu \mathrm{m}$, in hypermetropic and astigmatic group respectively). In contrast, myopic amblyopic subgroup showed a significantly thinner 
peripapillary RNFL in the amblyopic eyes than their fellow eyes, mean was $81.33 \pm 23.35$ and $110.00 \pm 17.58$ $\mu \mathrm{m}$ in amblyopic and fellow eyes, respectively. The study carried out by $\mathrm{Wu}$ and colleagues included 72 children aged 5-16 years with hyperopic anisometropic amblyopia, and detected that the mean peripapillary RNFL thickness in the amblyopic eyes (113.9 \pm 7.2$)$ was significantly thicker than that of the fellow eyes $(109.2 \pm 6.9 \mu \mathrm{m})$. The current study corroborated with two other studies by Tekin et al. [18] and Abdulghaffar et al. [16] , which have documented a thinner RNFL in myopic amblyopic eyes than their fellow eyes.

In this study, when comparing amblyopic eyes with their fellow eyes according to SE refractive error regarding CMT, only myopic amblyopic eyes yielded a significant thicker CMT in amblyopic eyes than that of fellow eyes, mean CMT was 199.00 \pm 26.851 and $191.00 \pm 26.00 \mu \mathrm{m}$ in amblyopic and fellow eyes, respectively.

Pang and colleagues reported the same result. They found a thicker CMT in the amblyopic eyes $(203.58 \pm 18.33 \mu \mathrm{m})$ of 31 patients compared to their normal fellow eyes $(191.00 \pm 15.76 \mu \mathrm{m})$ [19]. Zhao and colleagues investigated changes in macular thickness associated with high myopia and reported that with an increase in myopia degree, CMT increased [20]. This can be attributed to the vitreous traction on fovea due to stretching of the sclera as a result of elongation of axial length in highly myopic eyes [21].

In contrast to our result in the current study, Singh et al. [9] and Abdulghaffar et al. [16] reported no significant difference in CMT of the amblyopic eyes in any refractive subgroup (hypermetropia, myopia, and astigmatism) compared with their fellow eyes.

$\mathrm{B}$ applying further analysis in the present study to compare GCL+ thickness in anisometropic amblyopic eyes versus the fellow eyes according to SE refractive error, we did not find any significant difference between amblyopic and fellow eyes in any of anisometropic subgroups. The current study was consistent with the study carried out by Abdulghaffar and colleagues [16]. They did not report any significant difference between amblyopic and fellow eyes in any anisometropic subgroups. Mean GCL+ in amblyopic and fellow eyes in hypermetropic, myopic, and astigmatic subgroups were $80.85 \pm 3.48$ and $80.85 \pm 3.48,80.50 \pm 7.14$ and $80.50 \pm 4.89$, $81.36 \pm 4.31$ and $80.84 \pm 5.76 \mu \mathrm{m}$ in three subgroups, respectively.

The discrepancy in results of those mentioned previously studies may be attributed to the study design like the retinal structures that were measured and the different versions of OCT devices that were used for the studies and the use of different algorithms between OCT devices. Other factors which affected the OCT measurements presumably were the different patient's age, different number of participants, and biodiversity in races [8].

The limitations of this study was a relatively small sample size, which restrict the classification of the participants into subgroups according to the age, gender, severity of amblyopia (mild, moderate, or severe). We couldn't also divide the participants into persistent and recovered amblyopia. Therefore, further studies with larger number of amblyopic patients are needed.

The relatively broad age range (8-30 years old), and inclusion of normal control non-amblyopic subjects in the present study are considered the points of strength because the amblyopic patients may have similar but less severe abnormalities in the fellow eyes.

\section{Conclusion}

The measured OCT parameters including peripapillary RNFL thickness, macular parameters including (CMT, average macular thickness, and macular volume), and GCL+ thickness showed no significant differences in amblyopic eyes (group A) as compared to normal fellow eyes (group B) and normal eyes of nonamblyopic subjects (group C) .

However, by further secondary analysis to compare subgroups according to SE refractive error, peripapillary RNFL thickness revealed a significant increase in hypermetropic and astigmatic amblyopic eyes compared with their fellow eyes but it was significantly thinner in myopic amblyopic eyes than their fellow eyes. CMT revealed a significant increase in myopic amblyopic eyes only compared with their fellow eyes. Other OCT parameters did not show a significantly difference between amblyopic and their fellow eyes in different subgroups.

However, more studies with greater number of patients are required to confirm these findings.

\section{References}

[1] K. Ding, Y. Liu, X. Yan, X. Lin, T. Jiang. "Altered functional connectivity of the primary visual cortex in subjects with amblyopia." Neural Plasticity, Vol. 2013, PP.1-8, 2013.

[2] O. Joly, E. Frankó. "Neuroimaging of amblyopia and binocular vision: a review." Frontiers in Integrative Neuroscience, Vol. 8: PP.62, 2014.

[3] B.S. Goel. "Amblyopia." Clinical ophthalmology. In A.K. Gupta and V. Krishna, eds, Ninth ed. India: Elsevier Health Sciences, PP.216, 2012.

[4] A.M. Ansons, H. Davis. "Amblyopia." Diagnosis and management of ocular motility disorders. Fourth ed. India: Wiley Blackwell; PP.285-287, 2014.

[5] A. Bashinsky, E.K. Isbey, D.J. Browning, B.D. Fouraker, S.J. Grosser, S.R. Klapper, J.A. Savage, M.S. Ying: "Amblyopia". Basic and Clinical Science Course (BCSC), Section 6: Pediatric ophthalmology and strabismus. USA: American Academy of Ophthalmology, PP.79-86, 2019.

[6] R.K. Murthy, S. Haji, K. Sambhav, S. Grover, K.V. Chalam. "Clinical applications of spectral domain optical coherence tomography in retinal diseases." Biomedical Journal; Vol. 39 (2), PP.107-110, 2016.

[7] B.Z. Wang and D. Taranath. "A comparison between the amblyopic eye and normal fellow eye ocular architecture in children with hyperopic anisometropic amblyopia." J.,AAPOS: the official publication of the American Association for Pediatric Ophthalmology and Strabismus; Vol. 16, pp.428-430, 2012.

[8] P.G. Firat, E. Ozsoy, S. Demirel, T. Cumurcu and A. Gunduz. "Evaluation of peripapillary retinal nerve fiber 
layer, macula and ganglion cell thickness in amblyopia using spectral optical coherence tomography." International J.,Ophthalmology, Vol. 6, PP.90-94, 2013.

[9] N. Singh, J. Rohatgi, V.P. Gupta and V. kumar. "Measurement of peripapillary retinal nerve fiber layer thickness and macular thickness in anisometropia using spectral domain optical coherence tomography: a prospective study." Clinical Ophthalmology, Vol. 11, pp.429-434, 2017.

[10] M. Atakan, Ş. Çulfa, Ü. Çallı, A. D. Penbe, T. G. Atakan and Y. Özertürk. "Evaluation of retinal nerve fiber layer and macular thickness in amblyopia." J.,Clinical and Experimental Ophthalmology, Vol.6, PP.437, 2015.

[11] C.E. Al-Haddad, G. M. E. L. Mollayess, C.G. Cherfan, D. F. Jaafar and Z. F. Bashshur. "Retinal nerve fibre layer and macular thickness in amblyopia as measured by spectral-domain optical coherence tomography." The British J.,Ophthalmology, Vol. 95, PP.1696-1699, 2011.

[12] A.G. Alotaibi and B.A. Enazi. "Unilateral amblyopia: Optical coherence tomography findings." Saudi J.,Ophthalmology; Vol. 25, PP.405-409, 2011.

[13] S. Araki, A. Miki, T. Yamashita, K. Goto, K. Haruishi, Y. Ieki and A. Kiryu. "A comparison between amblyopic and fellow eyes in unilateral amblyopia using spectral-domain optical coherence tomography." Clinical Ophthalmology; Vol. 2014, PP.2199-2207, 2014.

[14] M.A. El-Hifnawy, A.F. Abo-Elkheir, A.A. Abo-Samra and K.A. Mohamed. "Spectral domain optical coherence tomography measurements in amblyopic Egyptian patients." Delta J.,Ophthalmology, Vol. 18: PP.26-31, 2017.

[15] M.A. Kasem and A.E. Badawi. "Changes in macular parameters in different types of amblyopia: optical coherence tomography study." Clinical Ophthalmology, Vol. 11: ,PP.1407-1416, 2017.

[16] A.T.T. Abdulghaffar, A. Al-Saadany and A.M. Ibrahim. "Optical coherence tomography-based comparison of retinal nerve fiber layer thickness and macular thickness in amblyopic and fellow eyes." Delta J.,Ophthalmology, Vol. 2019, PP.74-81, 2019.

[17] K.A. Park, D.Y. Park and S.Y. Oh. "Analysis of spectraldomain optical coherence tomography measurements in amblyopia: a pilot study." The British J.,Ophthalmology, Vol. 95, PP.1700-1706, 2011.

[18] K. Tekin, V. Cankurtaran, M. Inanc, M.A. Sekeroglu and P. Yilmazbas. "Effect of myopic anisometropia on anterior and posterior ocular segment parameters." International J.,Ophthalmology, Vol. 37, PP.377-384, 2016.

[19] Y. Pang, G. W. Goodfellow, C. Allison, S. Block and K. A. Frantz. "A prospective study of macular thickness in amblyopic children with unilateral high myopia. "Investigative and Ophthalmology Visual Science, Vol. 25, PP.2444-2449, 2011.

[20] Z. Zhao, X. Zhou, C. Jiang and X. Sun. "Effects of myopia on different areas and layers of the macula: a fourier-domain optical coherence tomography study of a Chinese cohort." BMC ophthalmology, Vol. 15, PP.1590, 2015.

[21] N.E. Samuel and S. Krishnagopal. "Foveal and macular thickness evaluation by spectral OCT and its relation with axial length in various degree of myopia." J.,Clinical and Diagnostic Research, Vol. 9, PP.1-4, 2015. 